\title{
Nur Gewinner
}

\section{Christoph Bosshard}

Dr. med., Vizepräsident der FMH, Departementsverantwortlicher DDQ

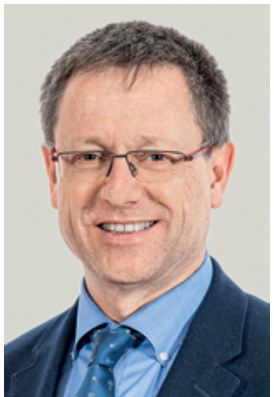

Die Verordnung über die Integrität und Transparenz im Heilmittelbereich VITH (www.fmh.ch/dienstleistungen/recht/vith.cfm) verpflichtet Leistungserbringer seit 1. Januar 2020 zur Weitergabe von allfällig ausgehandelten Rabatten an die Versicherten. Was aber soll die Leistungserbringer motivieren, Rabatte auszuhandeln, welche sie dann gar nicht behalten dürfen? Die Lösung liegt in den erwähnten Bestimmungen des Krankenversicherungsgesetzes (KVG). Diese sehen vor, dass Vergünstigungen gemäss Art. 56 Abs. 3 Bst. b KVG auf vertraglicher Basis nicht mehr vollumfänglich, sondern «mehrheitlich» von den Leistungserbringern und/oder Leistungserbringerorganisationen an die Schuldner der Vergütung weitergegeben werden müssen. Nicht weitergegebene Vergünstigungen müssen zur nachweislichen «Verbesserung der Qualität der Behandlung» der Patienten eingesetzt werden. Nach einer Phase von befristeten Vertragswerken ist es den Vertragspartnern HSK, CSS, Tarifsuisse und FMH gelungen, einen nationalen Vertrag betreffend die nicht vollständige Weitergabe von Vergünstigungen gemäss Art. 56 Abs. 3bis KVG abzu-

\section{Maximal 49 Prozent der erhaltenen Vergünstigungen können für Qualitäts- massnahmen eingesetzt werden.}

schliessen. Dieser bietet nun die Möglichkeit, mittels Anschlussverträgen maximal 49 Prozent der erhaltenen Vergünstigungen für Qualitätsmassnahmen einzusetzen. Denn Qualitätsarbeit kostet Geld. Während die Sicherung der notwendigen Qualität impliziter Bestandteil der Leistungserbringung ist, steigen fortwährend die Auflagen betreffend deren Dokumentation und Nachweis. Der Ruf nach Registern zum Beispiel ist allgegenwärtig. Auch wenn diese, vorausgesetzt sie werden richtig betrieben, Mehrwerte generieren, bleibt auf der Kostenseite ein Budgetposten, welcher bedient werden will. FMH-Empfehlungen und Informationen zu Registern finden Sie hier: www.fmh.ch/ themen/qualitaet-saqm/register.cfm. Aber entscheidend ist, was wir denn schliesslich mit den Registerdaten machen. Sie müssen ausgewertet werden und sollen uns schliesslich in der datenbasierten Qualitätsentwicklung unterstützen, also von der Themensetzung

\section{Die Hälfte der Vergünstigungen fliesst} zurück an die Versicherer und entlastet somit die Prämienseite.

bei Fortbildungsveranstaltungen bis hin zur Anpassung von Leitlinien und Schulung derselben. Qualitätsarbeit benötigt auch das notwendige Fachwissen und Erfahrung, und diese sind bei unseren Mitgliedern vorhanden. Wenn nun die Finanzierung sichergestellt ist, kann auch die notwendige Zeit investiert werden.

Auch die Prämienzahlenden gewinnen, denn die andere Hälfte der Vergünstigungen fliesst zurück an die Versicherer und entlastet somit die Prämienseite. Alle sprechen von der Prämienlast. Mit dem nun erreichten nationalen Vertrag betreffend die nicht vollständige Weitergabe von Vergünstigungen gemäss Art. 56 Abs. 3bis KVG leisten die Vertragspartner einen aktiven Beitrag zur Reduktion der Prämienlast im KVG. Für die FMH ist es zentral, dass nun zuerst die Erfahrungen aus dieser Gesetzesanpassung wie auch die Erfahrungen aus den neuen gesetzlichen Bestimmungen, wie Art. 58 KVG zu Qualität und Wirtschaftlichkeit, und den neuen Zulassungsbestimmungen gemäss Art. 55 KVG zu evaluieren sind, bevor allfällige weitere neue Gesetzesrevisionen wie z.B. eine Zielvorgabe für das Kostenwachstum in der OKP beschlossen würden. Also gibt es nur Gewinner: Die Prämienzahlenden, die Leistungserbringer und am allerwichtigsten: unsere Patientinnen und Patienten. Ich danke allen, welche mitgeholfen haben, diese Lösung zu ermöglichen! 